\title{
The Chebyshev collocation method for finding the eigenvalues of fourth-order Sturm-Liouville problems
}

\author{
Rahmat Darzi ${ }^{1, a *}$, Bahram Agheli ${ }^{2, b}$ \\ ${ }^{1}$ Department of Mathematics, Neka Branch, Islamic Azad University, Neka, Iran \\ ${ }^{2}$ Department of Mathematics, Qaemshahr Branch, Islamic Azad University, Qaemshahr, Iran \\ a r.darzi@iauneka.ac.ir, ${ }^{b}$ b.agheli@qaemshahriau.ac.ir \\ *r.darzi@iauneka.ac.ir
}

Keywords: Fourth order Sturm-Liouville problem, Chebyshev collocation method, eigenvalues.

\begin{abstract}
In this work, we have suggested that the Chebyshev collocation method can be employed for detecting the eigenvalues of fourth-order Sturm-Liouville problems. Two examples are presented subsequently. Numerical eventuates indicate that the present method is accurate.
\end{abstract}

\section{Introduction}

The boundary value problems for ordinary differential equations have a notable role theoretically. Also, they have diverse applications. A great number of physical, biological and chemical phenomena, can be explained through using boundary value problems. In this paper, Chebyshev collocation method is used to acquire the solutions for the subsequent fourth order nonsingular Sturm-Liouville problems

$$
\left(q_{0}(x) y^{\prime \prime}(x)\right)^{\prime \prime}+\left(q_{1}(x) y^{\prime}(x)\right)^{\prime}+\left(\mu v(x)-q_{2}(x)\right) y(x)=0, \quad a<x<b,
$$

or

$$
y^{(4)}=F\left(y(x), y^{\prime}(x), y^{\prime \prime}(x), y^{\prime \prime \prime}(x), \mu\right)
$$

or

$$
y^{(4)}+p_{3}(x) y^{\prime \prime \prime}(x)+p_{2}(x) y^{\prime \prime}(x)+p_{1}(x) y^{\prime}(x)+(\mu w(x)-r(x)) y(x)=0
$$

with the four linearly independent homogeneous boundary conditions

$$
\sum_{k=0}^{3} \alpha_{i k} y^{(k)}(a)+\beta_{i k} y^{(k)}(b)=0 .
$$

It is significant to note that $y(x)$ is an accurate solution of (3) and (4).

It is not simple to find solutions for fourth order Sturm-Liouville or there are no accurate solutions of Sturm-Liouville problems. There can be various approximate methods. Eq.(1) is mostly defined as the metamorphose of an elastic beam under several boundary conditions [1-7]. For instance, if we use problem (1) in Lidstone boundary value conditions $\left(y(a)=y(b)=y^{\prime \prime}(a)=y^{\prime \prime}(b)=0\right)$, it is applied to model such phenomena as the deflection of elastic beam. Many studies have been conducted on the collocation method in recent writing about the numerical solution of boundary value problem [8-11]. elik [12-14] studied the corrected collocation method to give an approximate calculation of eigenvalues of Sturm-Liouville and periodic Sturm-Liouville problems by simplified Chebyshev series. 


\section{Chebyshev collocation method}

Fourth-order nonsingular Sturm-Liouville problems with variable coefficients are of the form

$$
\left(q_{0}(x) y^{\prime \prime}\right)^{\prime \prime}+\left(q_{1}(x) y^{\prime}\right)^{\prime}+\left(\mu w(x)-q_{2}(x)\right)=0, \quad a<x<b,
$$

where $q_{2}(x), q_{1}(x), q_{0}(x)$ and $w(x)$ are piecewise continuous functions with $q_{0}(x), w(x) \geq 0$. The most popular homogeneity boundary condition is

$$
\sum_{k=0}^{3} \alpha_{i k} y(a)+\beta_{i k} y(b)=0, \quad 1 \leq i \leq 4 .
$$

Because finite range $a \leq t \leq b$ can be converted to the basic range $-1 \leq x \leq 1$ with the change of variable $w=\frac{(b-a) x+b+a}{2}$, sans losing generality, we presume $-1 \leq x \leq 1$.

In this method, for ( 3 ) we offer an evident solution of the form

$$
y(x)=\frac{a_{0}}{2} T_{0}(x)+\sum_{i=1}^{N} a_{i} T_{i}(x), \quad-1 \leq x \leq 1,
$$

where $T_{i}(x) i=0,1, \ldots, N$ are the Chebyshev polynomials and $a_{i}$ are indeterminate parameters.

The suggested solution (5) can be explained in the matrix form as

$$
[y(x)]=T_{x} A,
$$

where

$$
T_{x}=\left[T_{0}(x), T_{1}(x), \ldots, T_{N}(x)\right], \quad \text { and }, \quad A=\left[\frac{a_{0}}{2}, a_{1}, \ldots, a_{N}\right]^{T} .
$$

Collocation points may be taken as

$$
x_{i}=\frac{\cos (N-j) \pi}{N}, \quad j=1,2, \ldots, N-1,
$$

which denominates the turning points. If we change the Chebyshev collocation points in (3), the subsequent matrix expression can be obtained:

$$
Y^{(4)}+\sum_{k=1}^{3} P_{k} Y^{k}+(\mu W-R) Y=\theta,
$$

or

$$
Y^{(4)}+\sum_{k=0}^{3} P_{k} Y^{(k)} Y=\theta
$$


where,

$$
\begin{aligned}
& P_{0}=\mu W-R=\left[\begin{array}{cclc}
\mu w\left(x_{1}\right)-r\left(x_{1}\right) & 0 & \ldots & 0 \\
0 & \mu w\left(x_{2}\right)-r\left(x_{2}\right) & \ldots & 0 \\
\vdots & \vdots & \ddots & \vdots \\
0 & 0 & \ldots & \mu w\left(x_{N-1}\right)-r\left(x_{N-1}\right)
\end{array}\right] \\
& P_{k}=\left[\begin{array}{cccc}
p_{k}\left(x_{1}\right) & 0 & \ldots & 0 \\
0 & p_{k}\left(x_{2}\right) & \ldots & 0 \\
\vdots & & \ddots & \vdots \\
0 & & \ldots & p_{k}\left(x_{N-1}\right)
\end{array}\right], \quad k=1,2,3, \quad \theta=\left[\begin{array}{c}
0 \\
0 \\
\vdots \\
0
\end{array}\right]
\end{aligned}
$$

and

$$
Y^{(k)}=\left[\begin{array}{c}
y^{(k)}\left(x_{1}\right) \\
y^{(k)}\left(x_{2}\right) \\
\vdots \\
y^{(k)}\left(x_{N-1}\right)
\end{array}\right], \quad k=0,1, \ldots, 4
$$

The $\mathrm{k} t h$ derivative of (5) with respect to $x$ can be written as

$$
Y^{(k)}\left(x_{j}\right)=\frac{a_{0}^{(k)}}{2} T_{0}\left(x_{j}\right)+\sum_{i=1}^{N} a_{i}^{(k)} T_{i}\left(x_{j}\right),
$$

where $a_{i}^{(k)}$ are Chebyshev coefficients $a_{i}^{(0)}=a_{i}$ and $y^{(0)}(x)=y(x)$. The $y^{(k)}\left(x_{j}\right)$ can be written in the matrix form as

$$
\left[y^{(k)}\left(x_{j}\right)\right]=T_{x_{j}} A^{(k)}, \quad k=0,1,2,3,4,
$$

or matrix equation

$$
Y^{(k)}=T A^{(k)}, \quad k=0,1,2,3,4
$$

where

$$
T=\left[\begin{array}{c}
T_{x_{1}} \\
T_{x_{2}} \\
\vdots \\
T_{x_{N-1}}
\end{array}\right]=\left[\begin{array}{cccc}
T_{0}\left(x_{1}\right) & T_{1}\left(x_{1}\right) & \ldots & T_{N}\left(x_{1}\right) \\
T_{0}\left(x_{2}\right) & T_{1}\left(x_{2}\right) & \ldots & T_{N}\left(x_{2}\right) \\
& & & \\
\vdots & & \ddots & \vdots \\
T_{0}\left(x_{N-1}\right) & T_{1}\left(x_{N-1}\right) & \ldots & T_{N}\left(x_{N-1}\right)
\end{array}\right], \quad A^{(k)}=\left[\begin{array}{c}
\frac{1}{2} a_{0}^{(k)} \\
a_{1}^{(k)} \\
\vdots \\
a_{N}^{(k)}
\end{array}\right]
$$

The relevance between the Chebyshev coefficient matrix $A$ of $y(x)$ and Chebyshev coefficient matrix $A^{(k)}$ of $Y^{(k)}$ can be illustrated as

$$
A^{(k)}=2^{k} M^{k} A,
$$


where

$$
M=\left[\begin{array}{cccccccccc}
0 & \frac{1}{2} & 0 & \frac{3}{2} & 0 & \frac{5}{2} & 0 & \frac{7}{2} & \cdots & \frac{N}{2} \\
& & 2 & 0 & 4 & 0 & 6 & 0 & \cdots & 0 \\
& & & 3 & 0 & 5 & 0 & 7 & \cdots & N \\
& & & & 4 & & 6 & 0 & \cdots & 0 \\
& & & & 5 & 0 & 7 & \cdots & N \\
& & & & \ddots & & 6 & 0 & \cdots & 0 \\
\vdots & & & & & & 7 & & \vdots \\
& & & & & & & & \\
& & & & & & & \ddots & \\
& & & & & & & & \\
0 & & & & & & & & N \\
& & & & \cdots & & & & 0
\end{array}\right]
$$

if $N$ is odd, and

$$
M=\left[\begin{array}{ccccccccccc}
0 & \frac{1}{2} & 0 & \frac{3}{2} & 0 & \frac{5}{2} & 0 & \frac{7}{2} & \cdots & \frac{N-1}{2} & 0 \\
& & 2 & 0 & 4 & 0 & 6 & 0 & & \cdots & 0 \\
& & & 3 & 0 & 5 & 0 & 7 & & \cdots & N \\
& & & & 4 & & 6 & 0 & & \cdots & 0 \\
& & & & & 5 & 0 & 7 & & \cdots & N \\
& & & & \ddots & & 6 & 0 & & \cdots & 0 \\
\vdots & & & & & & & 7 & & \vdots \\
& & & & & & & & & \\
& & & & & & & & \ddots & \\
& & & & & & & & & \\
& & & & & & & & & & \\
0 & & & & & & & & & & 0
\end{array}\right]
$$

if $N$ is even.

Accordingly, the matrix demonstration of (8) and the matrix equation (6) can be expressed in order as

$$
Y^{(k)}=2^{k} T M^{k} A, \quad \text { and } \quad\left[2^{4} T M^{4}+\sum_{k=0}^{3} P_{k} 2^{k} T M^{k}\right] A=\theta .
$$

Moreover, the boundary conditions can be written in the matrix form as

$$
\sum_{j=0}^{3} 2^{j}\left(\alpha_{i j} T_{-1}+\beta_{i j} T_{1}\right) M^{j} A=0, \quad i=1,2,3,4,
$$

on the interval $[-1,1]$, where

$$
T_{-1}=\left[1,-1,1,-1, \ldots,(-1)^{N}\right], \quad T_{1}=[1,1,1, \ldots, 1] .
$$


By using the subsequent display:

$$
\begin{aligned}
& W=\left[w_{i j}\right]=\sum_{k=0}^{3} P_{k} 2^{k} T M^{k}, \quad i=1,2,3, \ldots, N-1, \quad j=0,1,2, \ldots, N, \\
& U_{i}=\left[u_{i 0}, \ldots, u_{i N}\right]=\sum_{j=0}^{1} 2^{j}\left(\alpha_{i j} T_{-1}+\beta_{i j} T_{1}\right) M^{j}, \quad i=1,2,3,4,
\end{aligned}
$$

Matrix equation can be displayed as

$$
\widetilde{W} A=\theta
$$

where

$$
\widetilde{W}=\left[\begin{array}{cccc}
w_{10} & w_{11} & \ldots & w_{1 N} \\
w_{20} & w_{21} & \ldots & w_{2 N} \\
\vdots & \vdots & & \vdots \\
w_{N-10} & w_{N-11} & \ldots & w_{N-1 N} \\
u_{10} & u_{11} & \ldots & u_{1 N} \\
u_{20} & u_{21} & \ldots & u_{2 N} \\
u_{30} & u_{31} & \ldots & u_{3 N} \\
u_{40} & u_{41} & \cdots & u_{4 N}
\end{array}\right]
$$

The rank of the matrix $\widetilde{W}$ is $N+1$. This is a set of equations for the uncertain parameter $a_{i}^{k}$. This set of equations has a non obvious solution only if the determinant of the coefficients matrix vanishes. This gives an equation of degree $N-1$ in $\mu$ and has $N-1$ roots which are the first $N-1$ numerical eigenvalues of the main problem.

\section{Numerical results}

After applying the procedure described in previous section, we produce two numerical results of the fourth order Sturm-Liouville problem in this section.

Example 1. Assume the following fourth order eigenvalue problem

$$
y^{(4)}(x)=\mu y(x)
$$

subject to

$$
y(-1)=y^{\prime}(-1)=0 \quad y(1)=y^{\prime \prime}(1)=0 .
$$

We can choose the trial solution as

$$
y(x)=\frac{a_{0}}{2} T_{0}(x)+\sum_{i=1}^{5} a_{i} T_{i}(x), \quad-1 \leq x \leq 1,
$$

where $T_{i}(x), i=0,1, \ldots, N$ are the chebyshev polynomial and $a_{i}$ are non specified parameters. Through applying the chebyshev collocation method, points can be obtained as

$$
x_{j}=\cos \frac{(5-j) \pi}{5}, \quad j=1,2,3,4
$$


If we change the Chebyshev collocation points in (12), we will get the expression below

$$
y^{(4)}\left(x_{j}\right)-\mu y\left(x_{j}\right)=0,
$$

or it could be written in the matrix form

$$
Y^{(4)}-P Y=0
$$

where

$$
P=\left[\begin{array}{cccc}
-\mu & 0 & 0 & 0 \\
0 & -\mu & 0 & 0 \\
0 & 0 & -\mu & 0 \\
0 & 0 & 0 & -\mu
\end{array}\right]
$$

and

$$
Y^{(4)}=\left[\begin{array}{l}
y^{(4)}\left(x_{1}\right) \\
y^{(4)}\left(x_{2}\right) \\
y^{(4)}\left(x_{3}\right) \\
y^{(4)}\left(x_{4}\right)
\end{array}\right]
$$

The fourth order derivative of (14) with respect to $x$ may be written as

$$
y^{(4)}\left(x_{j}\right)=\sum_{i=0}^{6} a_{i}^{(k)} T_{i}\left(x_{j}\right)
$$

where $a_{0}^{(4)}=192 a_{4}, a_{1}^{(4)}=1920 a_{5}$ and $a_{i}^{(4)}=0, \quad i=2,3,4,5$.

Applying (11), we offer an equation of degree 4 in $\mu$ with four roots which are the first four approximate eigenvalues of the (12-13) i.e. $\mu_{i}=0, i=1,2,3$ and $\mu_{4}=0.00076$.

Example 2. Suppose the following fourth order eigenvalue problem

$$
y^{(4)}(x)=0.02 x^{2} y^{\prime \prime}(x)+0.04 x y^{\prime}(x)-(0.0001-0.02) y(x)+\mu y(x), \quad x \in(-1,1)
$$

subject to

$$
y(-1)=y^{\prime}(-1)=0 \quad y(1)=y^{\prime \prime}(1)=0 .
$$

We can choose the trial solution as

$$
y(x)=\frac{a_{0}}{2} T_{0}(x)+\sum_{i=1}^{5} a_{i} T_{i}(x), \quad-1 \leq x \leq 1,
$$

where $T_{i}(x), i=0,1, \ldots, N$ are presumed the Chebyshev polynomial and $a_{i}$ are undetermined parameters.

We apply the same method as we did in Example 1. Therefore, the first four approximate eigenvalues of the (15-17) are given in Table 1 .

Table 1: The first four eigenvalues for Example 2

\begin{tabular}{|c|c|}
\hline $\mathrm{i}$ & $\mu_{i}$ \\
\hline 1 & -383.2763 \\
2 & -77.41000 \\
3 & -0.138400 \\
4 & -0.260900 \\
\hline
\end{tabular}




\section{Conclusions}

In this work, we have used Chebyshev collocation method to acquire the eigenvalues of fourth-order Sturm-Liouville problems. The approximate examples used in this paper consequently display the efficiency of the present method. Also, the examples provided and all numerical calculations in the present study have been performed on a $P C$, applying programs written in Matlab.

$*$

\section{References}

[1] P. Amodio, S. Giuseppina, Reprint of Variable-step finite difference schemes for the solution of Sturm-Liouville problems, Communications in Nonlinear Science and Numerical Simulation, 21.1 (2015) 12-21.

[2] J. Schroder, Fourth order two point boundary value problems: Estimate by two side bounds, Nonlinear Anal, 8 (1984) 107-144.

[3] R.P. Agarwal, M.Y. Chow Iterative methods for a Fourth order boundary value problem, J. Comput. Appl. Math, 10 (1984) 203-217.

[4] R.P. Agarwal, On the fourth-order boundary value problems arising in beam analysis, Diff. int. Eq, 2 (1989) 91-110.

[5] D. O'Regan, Solvability of some fourth (and higher) order singular boundary value problems, J. Math. Anal. Appl, 161 (1991) 78-116.

[6] C. Gupta, Existence and uniquness theorems for a bending of an elastic beam equation at resonance, J. Math. Anal. Appl, 135 (1988) 208-225.

[7] L. Liu, X. Zhang, Y. Wu, Positive solutions of Fourth-order nonlinear singular Sturm-Liouville eigenvalue problems, J. Math. Anal. Appl, 326 (2007) 1212-1224.

[8] B. Chanane, Eigenvalues of fourth order Sturm-Liouville problems using Fliess series, Journal of Computational and Applied Mathematics, Volume 96, Issue 2, 15 September 1998, Pages 91-97, ISSN 0377-0427, http://dx.doi.org/10.1016/S0377-0427(98)00086-7.

[9] A.H. Saleh Taher, A. Malek, S.H. Momeni-Masuleh, Chebyshev differentiation matrices for efficient computation of the eigenvalues of fourth-order Sturm-Liouville problems, Applied Mathematical Modelling, Volume 37, Issue 7, 1 April 2013, Pages 4634-4642, ISSN 0307-904X, http://dx.doi.org/10.1016/j.apm.2012.09.062.

[10] R.D. Russel, L.F.Shmpine, A collocation method for boundary value problems, Numer. Math, 19 (1972) 1-28.

[11] R. Weiss, The application of implicit Runge-Kutta and collocation methods to boundary value problems, Math. Comput, 28 (1974) 449-464.

[12] I. elik, Approximate calculation of eigenvalues with the method of weighted residual collocation method, J. Comput. Appl. Math, 160 (2005) 401-410.

[13] I. elik, Approximate compution of eigenvalues with Chebyshev collocation method, J. Comput. Appl. Math, in press, doi:10.1016/j.amc.2004.08.024.

[14] I. elik, G. Gokmen Approximate solution of periodic Sturm-Liouville problems with Chebyshev collocation method, J. Comput. Appl. Math, in press, doi:10.1016/j.amc.2004.11.038. 